\title{
Fourier Analysis of Radio Bursts Observed with Very High Time Resolution
}

\author{
B.P. Dąbrowski • M. Karlický • P. Rudawy
}

Received: 26 November 2013 / Accepted: 3 September 2014 / Published online: 7 October 2014

(C) The Author(s) 2014. This article is published with open access at Springerlink.com

\begin{abstract}
Numerous solar radio bursts were observed in 2000-2001 using the Torun radio spectrograph with its unique time resolution of 80 microseconds. This high time resolution enables an in-depth analysis of the time evolution of the power spectra and Fourier spectral indices of selected short radio bursts. We analyze the power-spectrum parameters and variability for two millisecond radio dm-spike events and one drifting pulsation structure (DPS) event, for which the structures of the recorded signals were analyzed with an effective time resolution of $0.0008 \mathrm{~s}$ in five adjacent frequency bands of their radio spectra.

We found that Fourier spectral indices varied rapidly when radio fine structures were recorded. Otherwise they remained close to zero, indicating the presence of noise. For the $10-40 \mathrm{~Hz}$ frequency interval of the power spectra the mean values of the Fourier spectral indices varied up to -1.51 for the dm-spikes and up to -1.53 for the DPS event. Crosscorrelation coefficients of radio fluxes in adjacent frequency bands varied between 0.12 and 0.60 for $\mathrm{dm}$-spikes and between 0.77 and 0.94 for the DPS event. The shortest fine structure found in the studied events lasted $0.001 \mathrm{~s}$.

These results are entirely new for dm-radio spikes and confirm a single previous result found for the DPS events. On the basis of our results, we propose that dm-spikes and DPS events are physically similar and that both are signatures of cascades of interacting plasmoids of different sizes.
\end{abstract}

New Eyes Looking at Solar Activity: Challenges for Theory and Simulations

Guest Editors: Silja Pohjolainen and Marian Karlický

B.P. Dąbrowski · M. Karlický

Astronomical Institute, Academy of Sciences of the Czech Republic, Fričova 298, 25165 Ondřejov, Czech Republic

B.P. Dąbrowski

e-mail: bdabrow@asu.cas.cz

M. Karlický

e-mail: karlicky@asu.cas.cz

P. Rudawy (凶)

Astronomical Institute, University of Wrocław, ul. Kopernika 11, 51-622 Wrocław, Poland

e-mail: rudawy@astro.uni.wroc.pl 
Keywords Solar corona $\cdot$ Flares $\cdot$ Radio bursts

\section{Introduction}

Diverse solar radio bursts are classified into radio bursts of types I, II, III, IV, and V. The type IV radio bursts are especially rich in fine structures such as pulsations, spikes, fibres, zebras, and other. The analysis of radio spectra of these fine structures with high time resolution always raises the question whether even shorter fine structures are generated as well. Therefore, it is a common endeavor to increase the time resolution of dynamical radio spectra as much as possible. Currently, the time resolution of solar radio spectrographs is in the range from 1 to $0.001 \mathrm{~s}$, except for the Torun radio spectrograph, which was used for solar observations during the years 2000 and 2001 with an unprecedented time resolution of 80 microseconds.

We used the high time resolution solar observations collected with the Torun radio spectrograph to search for short fine structures in radio bursts. For this purpose we developed a method based on the Fourier transform, which we applied to three selected events that included fine structures. We investigated two examples of millisecond radio dm-spikes (dmspikes) and one example of a drifting pulsation structure (DPS). This selection was made because we expected that these fine structures might be caused by similar processes occurring on very short time-scales.

The $\mathrm{dm}$-spikes are observed in the radio spectrum in clouds of up to $10^{4}$ individual spikes. They are characterized by very high brightness temperatures $\left(T_{\mathrm{b}} \approx 10^{15} \mathrm{~K}\right)$ and short durations ( $\leq 0.1 \mathrm{~s})$ (Benz, 1986). Their observational parameters have been described in several papers, e.g., in Mészárosová et al. (2003). As shown by Karlický, Sobotka, and Jiřička (1996) and by Karlický, Jiřička, and Sobotka (2000), Fourier spectra of the spikes have a power-law form and show that there is no characteristic frequency bandwidth of the spikes. To our knowledge, there are no Fourier analyses published of the evolution of the spikes with time. Theoretical models for spikes can be divided into two groups: a) those based on the plasma emission and acceleration processes (Kuijpers, van der Post, and Slottje, 1981), and b) those based on the electron-cyclotron maser (Fleishman and Yastrebov, 1994). It was recently proposed that these spikes are generated during fragmented magnetic reconnection process in solar flares (Karlický, Bárta, and Jiřička, 2013).

Drifting pulsating structures are observed during the impulsive phases of strong eruptive flares in association with plasmoid ejections (Kliem, Karlický, and Benz, 2000; Karlický, 2004). Usually, they slowly drift toward lower frequencies and are composed of many fast-drifting narrowband type III-like bursts. Observational details of these DPS were described by e.g., Kundu et al. (2001), Khan et al. (2002), Karlický, Fárník, and Mészárosová (2002), Karlický et al. (2005), and Benz, Battaglia, and Vilmer (2011). The first theoretical model of DPS was proposed by Kliem, Karlický, and Benz (2000). They assumed that each individual burst of the slowly drifting pulsating structure was generated by a beam of suprathermal electrons, accelerated during a peak of the electric field in the quasiperiodic regime of magnetic field reconnection. These electron beams are trapped inside the plasmoid, where they generate Langmuir (longitudinal plasma) waves. Then the Langmuir waves are converted into electromagnetic (radio) waves. A limited range of plasma densities in the plasmoid limits the range of the emission frequencies. The global slow negative drift of the DPS is explained by an expansion of the plasmoid during its upward propagation in the solar corona toward lower plasma densities.

However, as was shown in several theoretical papers (Tanuma et al., 2001; Shibata and Tanuma, 2001; Bárta et al., 2011a, 2011b; Karlický and Bárta, 2011; Karlický, Bárta, and 
Nickeler, 2012), reconnection processes in the flare current sheet lead to its fragmentation, i.e., to the formation of a cascade of plasmoids in a turbulent regime. Thus, it is highly probable that in addition to a dominant plasmoid generating DPS, there are many small plasmoids of short lifetime in its vicinity. Therefore, a Fourier analysis of DPS might reveal short processes. So far, only one DPS, observed during the 12 April 2001 flare at $1420 \mathrm{MHz}$ and $610 \mathrm{MHZ}$ frequencies by the Trieste radio polarimeters with $1 \mathrm{~ms}$ time resolution, has been analyzed by a similar method (Karlický et al., 2005). In this case, the Fourier spectra had a power-law form with a power-law index in the range -1.3 to -1.6 .

In the present article we not only extend the study of DPS, we also analyze for the first time two events with dm-spikes and compare our results. Also for the first time, we are able to investigate the selected fine structures not only with a uniquely high time resolution, but also simultaneously in numerous adjacent frequency channels.

\section{Observational Data}

The high time resolution radio spectra of the investigated events were collected with the $15 \mathrm{~m}$ radio telescope of the Torun Centre for Astronomy, Nicolaus Copernicus University in Torun, Poland (located in Piwnice) and the high time resolution multichannel radio spectrograph Penn State Pulsar Machine II (PSPM II) (Cadwell, 1997). The radio spectrograph was built mainly for observations of radio pulsars. However, from February 2000 until December 2001 it was used with great success for solar observations. Radio spectra of the solar events were recorded in the $1352-1490 \mathrm{MHz}$ frequency band split into 46 adjacent frequency bands of $3 \mathrm{MHz}$ width each, with an unprecedented high time resolution of 80 microseconds. A total observing time of 1990.4 hours covered the 357 observing days. The primary aim of these observations was studying radio spectra of dm-spikes, but various other phenomena, including numerous radio pulsations and drifting structures were also recorded. In-depth analyses of the variability and spectral properties of the observed dm-spikes were published by Dąbrowski et al. (2005) and Dąbrowski, Rudawy, and Karlický (2011).

Solar radio emission is also routinely obtained at Ondřejov Observatory with the $10 \mathrm{~m}$ radio telescope connected to a $0.8-2.0 \mathrm{GHz}$ swept-frequency broad-band radio spectrograph. The whole observed $0.8-2.0 \mathrm{GHz}$ band is divided into 256 frequency channels with bandwidths of $3 \mathrm{MHz}$ each, set $4.69 \mathrm{MHz}$ apart. The temporal resolution of the collected radio spectra of analyzed events was equal to 0.1 s (Jiřička et al., 1993; Karlický, Jiřička, and Sobotka, 2000).

After carefully inspecting the available data, we selected three radio events for a detailed analysis. Two events include dm-spikes observed with the Torun radio spectrograph on 15 March 2000 and 21 June 2000 and one event includes DPS event observed on 12 July 2000 simultaneously with radio spectrographs in Toruń and in Ondřejov.

\subsection{Radio dm-spike Event on 15 March 2000}

The dm-spikes were recorded between 12:20:06 UT and 12:21:08 UT (Figure 1) during the DCIM (decimeter, complex, highly structured radio emission) event, observed by us at Torun from 12:19:50 UT (the emission started before the beginning of our observations). The event peaked at 12:20:50 UT and ended at 12:26:22 UT (Figure 2, upper panel). The dm-spikes occurred during the impulsive phase of the C5 GOES-class solar flare in Active Region NOAA 8906 (S18W19). 


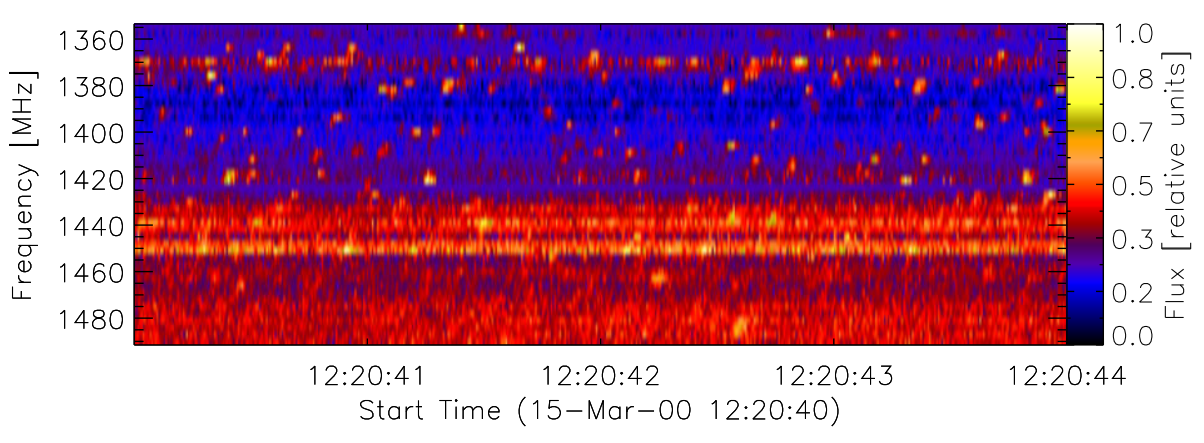

Figure 1 The dm-spikes recorded at Toruń on 15 March 2000.

\subsection{Radio dm-spike Event on 21 June 2000}

Numerous dm-spikes were recorded between 09:24:59 UT and 09:26:05 UT (Figure 3) during the long maximum of the radio burst, which was observed at Torun between 09:24:00 UT and 09:30:08 UT on 21 June 2000 (Figure 4, upper panel). The dm-spikes occurred at the end of the impulsive phase of the M1.3 GOES-class solar flare, which started at 09:22 UT, reached maximum at about 09:29 UT, and ended at 09:37 UT. The flare occurred in Active Region NOAA 9042 (N24W34).

\subsection{Radio DPS Event on 12 July 2000}

The radio event was observed at Ondřejov between 10:28 UT and 10:56 UT and at Torun between 10:30:50 UT and 10:39:54 UT (Figures 5 and 6, upper panel). The DPS occurred during the impulsive phase of the X1.9 GOES-class solar flare (which started at 10:18 UT) in Active Region NOAA 9077 (N18E19). It was recorded in the entire available frequency range $(0.8-2.0 \mathrm{MHz})$ of the Ondřejov radio spectrograph.

\section{Data Analysis}

The high time resolution raw radio data were collected at Torun with the PSPM II multichannel radio spectrograph in 46 adjacent frequency bands with a time resolution of $80 \mathrm{mi}-$ croseconds. The effective time resolution of the data was limited to $0.0008 \mathrm{~s}$, however, to increase the signal-to-noise $(\mathrm{S} / \mathrm{N})$ ratio.

While the investigated individual emissions were very short, the whole data set was divided into separate eight-second-long intervals, long enough to allow the spectral analysis of the short fine structures (Figures 2, 4, and 6, upper panels). For each time interval the standard Fourier analyses of the signals were performed in five adjacent frequency channels, where the short emissions were present and had the highest $\mathrm{S} / \mathrm{N}$ ratio, selected from all the channels of the recorded radio spectrogram. The power spectra of the signals were calculated in a standard way using a Fast Fourier Transform procedure (Figure 7 shows three examples of the power spectra).

Fourier spectral indices of the power spectra and cross-correlation coefficients were calculated for all the analyzed frequency bands. Fourier spectral indices were evaluated as the slopes of the linear fits to the power spectra in an arbitrary selected $10-40 \mathrm{~Hz}$ frequency band of the spectra for all investigated time intervals and bands (Figure 7). 

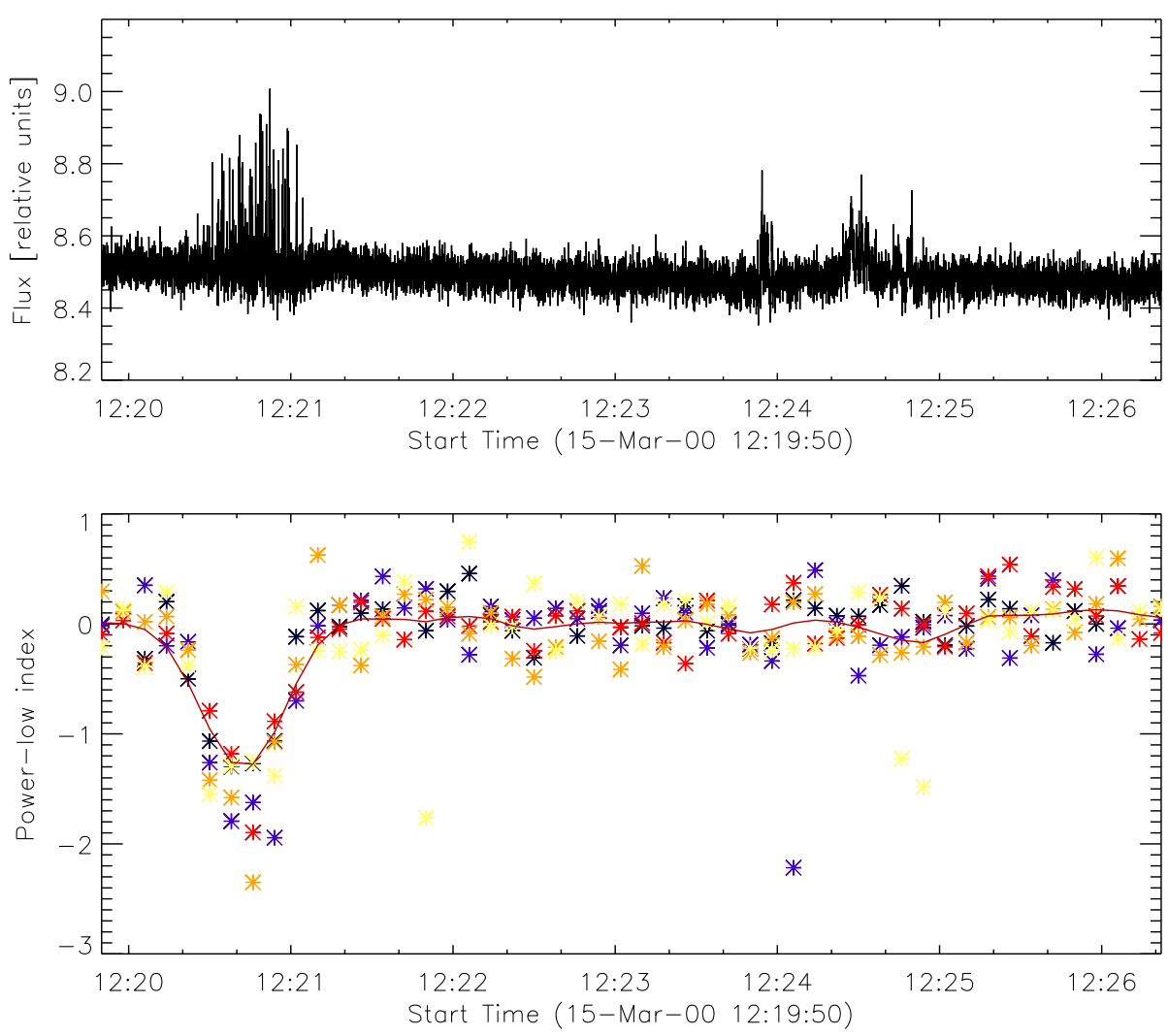

Figure 2 Radio event with dm-spikes recorded at Torun on 15 March 2000 between 12:19:50 UT and 12:26:22 UT with an effective time resolution of $0.0008 \mathrm{~s}$ (upper panel). The dm-spikes occurred between 12:20:06 UT and 12:21:08 UT. The Fourier spectral indices of the power spectra were calculated for five adjacent frequency bands extending between $1401.5 \mathrm{MHz}$ and $1413.5 \mathrm{MHz}, 3 \mathrm{MHz}$ wide each (shown with colored stars). The power spectra were calculated for eight-second-long consecutive cuts of the signal, the Fourier spectral indices were only determined for a $10-40 \mathrm{~Hz}$ band of the power spectra. The red line shows double-smoothed values of momentary average Fourier spectral indices (lower panel).

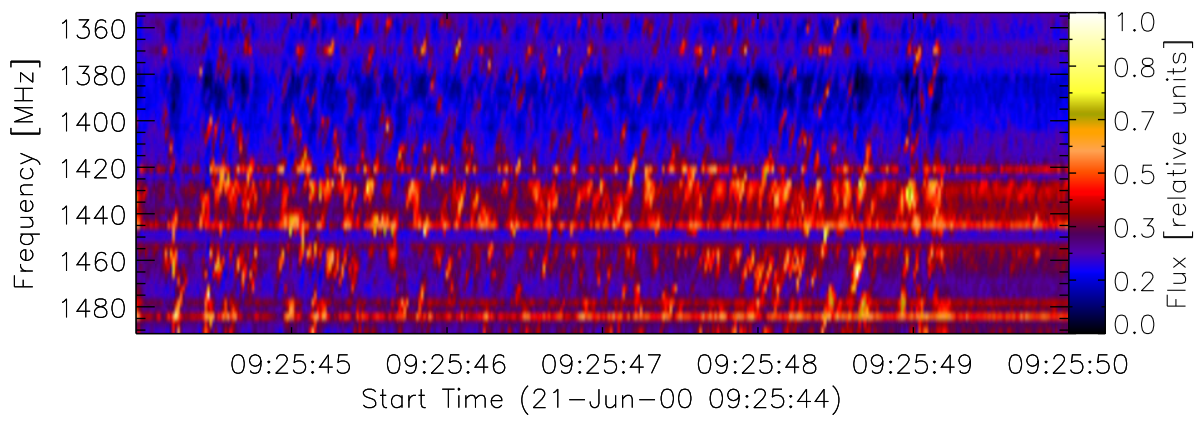

Figure 3 The dm-spikes recorded at Toruń on 21 June 2000. 

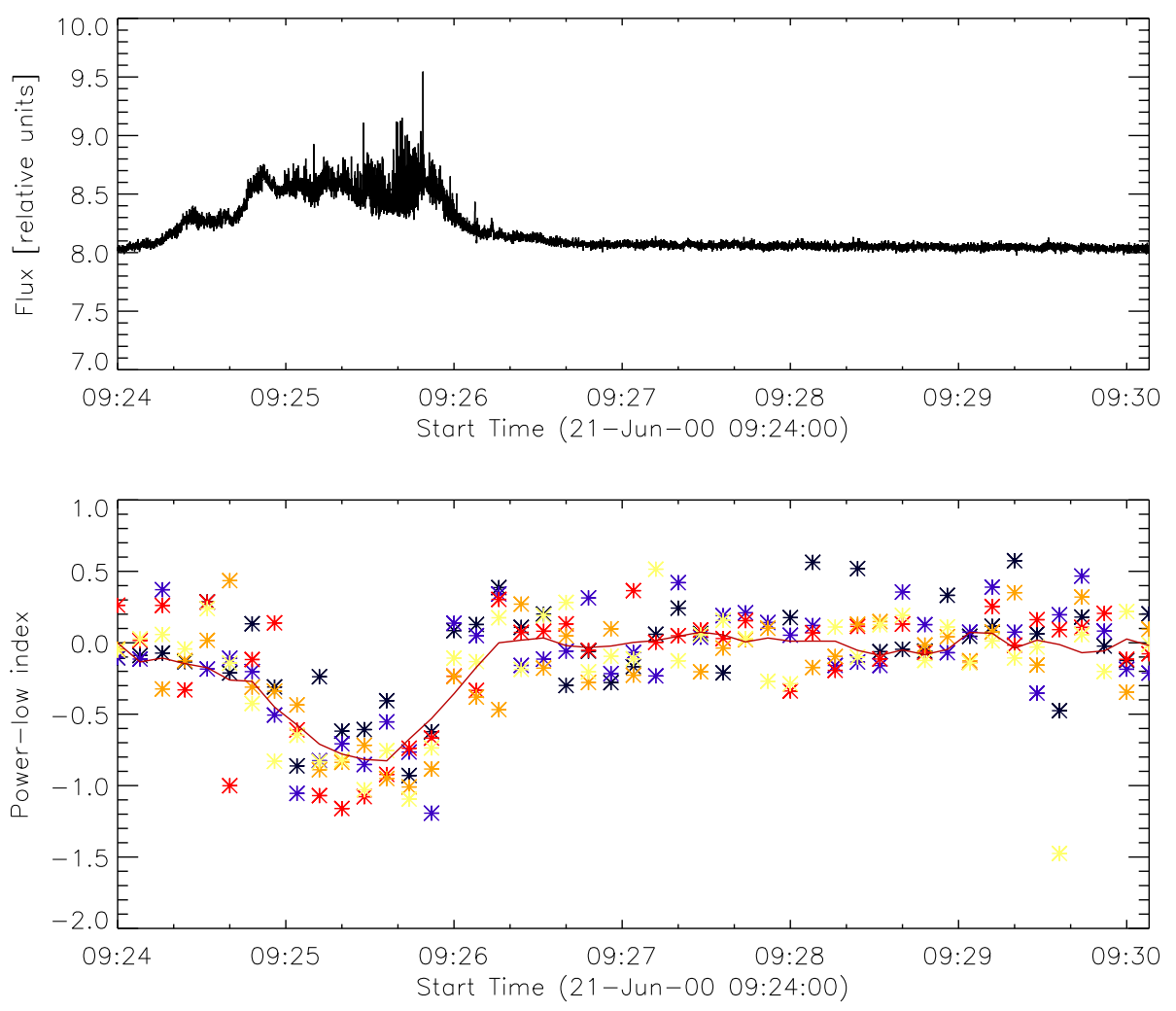

Figure 4 Radio event with dm-spikes recorded at Toruń on 21 June 2000 between 09:24:00 UT and 09:30:08 UT (upper panel). The dm-spikes occurred between 09:24:59 UT and 09:26:05 UT. The Fourier spectral indices of the power spectra calculated for five adjacent frequency bands extending between 1425.5 MHz and 1437.5 MHz, $3 \mathrm{MHz}$ wide each (shown with colored stars). The red line shows doublesmoothed values of momentary average Fourier spectral indices (lower panel).

\section{Results}

We analyze two radio events that include dm-spikes observed on 15 March 2000 and 21 June 2000 in the $1352-1490 \mathrm{MHz}$ band observed at Torun Observatory with an effective time resolution of $0.0008 \mathrm{~s}$ and one DPS event on 12 July 2000 observed simultaneously in the decimeter band $(0.8-2.0 \mathrm{GHz})$ at Ondřejov and in the $1352-1490 \mathrm{MHz}$ band at Torun (Figures 2, 4, and 6, upper panel). Figure 7 shows three examples of the power spectra of the eight-second-long data sets that include the investigated radio events.

The very high time resolution of the data enables us to compute not only individual Fourier spectra, but also their time evolution, as shown in Figures 2, 4, and 6 (lower panels). Although the values of the Fourier spectral indices from different channels are scattered, a general trend in their evolution is clearly visible. At times out of the cloud of dm-spikes and DPS, the values of the Fourier spectral indices were close to zero, indicating the presence of noise. Nonzero values of the Fourier spectral indices were found only during dm-spikes and DPS. For the $10-40 \mathrm{~Hz}$ frequency interval of the power spectra, mean Fourier spectral indices, defined arbitrarily as the mean values of the momentary Fourier spectral indices calculated for all the investigated adjacent frequency bands of the radio spectrogram, varied 

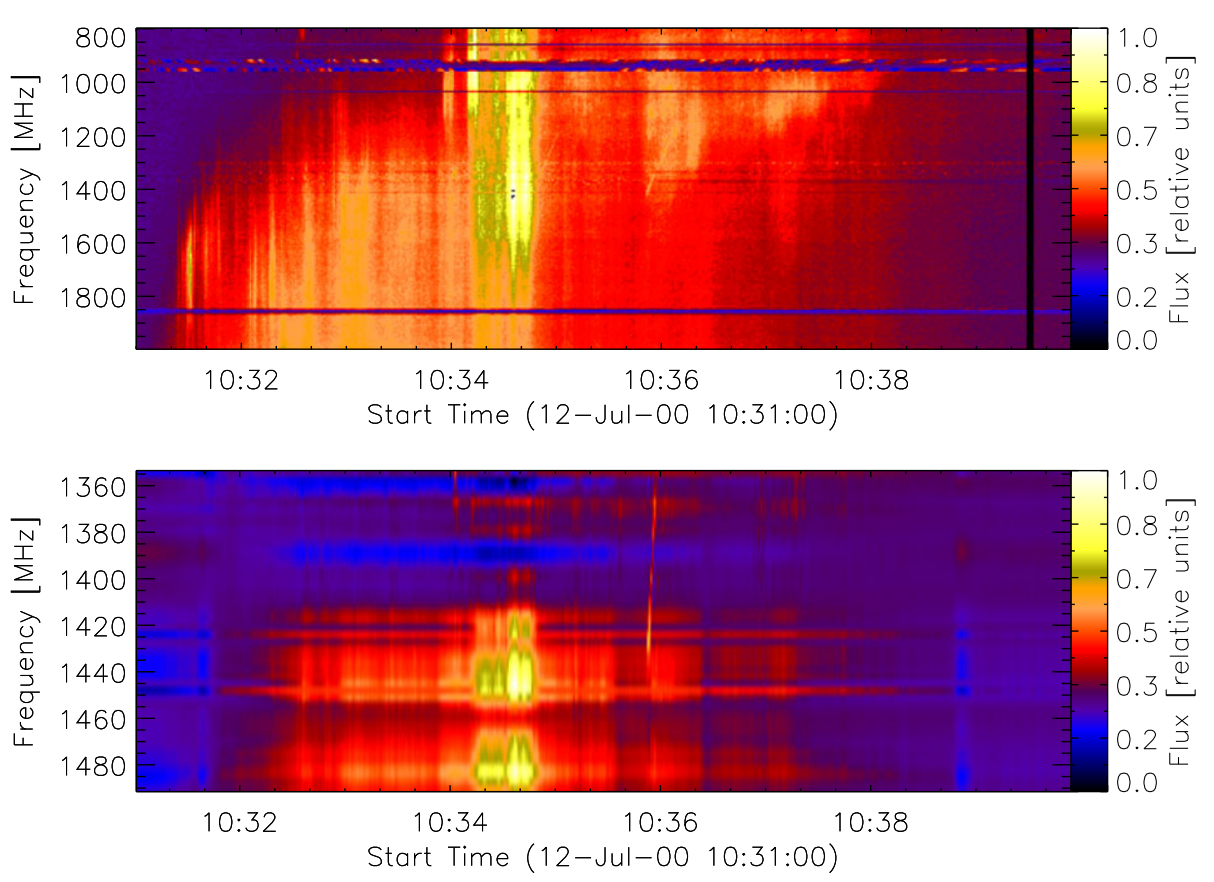

Figure 5 Drifting pulsating structure recorded simultaneously at Ondřejov (upper panel) and Toruń (lower panel) on 12 July 2000.

from zero to -1.51 for $\mathrm{dm}$-spikes recorded on 15 March 2000, from zero to -0.91 for the $\mathrm{dm}$-spikes recorded on 21 June 2000, and from zero to -1.53 for DPS event on 12 July 2000. However, the lowest and highest values of the Fourier spectral indices of the individual frequency bands (shown with colored stars in Figures 2, 4, and 6) obviously varied more strongly, up to -2.35 for the dm-spikes recorded on 15 March 2000, up to -1.5 for the dm-spikes recorded on 21 June 2000, and up to -1.85 for the DPS event on 12 July 2000. In the lower panels of Figures 2, 4, and 6, thin red lines show double-smoothed values of the momentary average Fourier spectral indices. They show time variations of the mean indices, where the applied box-car window was three points wide for the 15 March 2000 data and five points wide for the 12 July 2000 and 21 June 2000 data.

All spectra show a relatively flat low-frequency part, while in the range $10-40 \mathrm{~Hz}$ the spectrum has a power-law form $\left(\sim f^{-\gamma}\right)$. Most of the power spectra are flat at the highest frequencies (above about $40 \mathrm{~Hz}$ ) as a result of noise, but for many spectra the power-law parts extended to frequencies higher than $40 \mathrm{~Hz}$.

During the dm-spike event recorded on 15 March 2000 between 12:19:50 UT and 12:26:22 UT, the cross-channel correlation coefficients varied between 0.12 and 0.15 ; while for the dm-spike event on 21 June 2000 recorded between 09:24:00 UT and 09:30:08 UT, they varied between 0.50 and 0.60 . However, during the DPS event on 12 July 2000 between 10:30:50 UT and 10:39:54 UT, the cross-channel coefficients reached values between 0.77 and 0.94 . The relatively low values of the cross-channel correlation coefficients for $\mathrm{dm}$-spikes arise because their frequency bandwidths are more narrow than those of the DPS.

We also searched for the shortest fine structures in the studied events. Examples recorded during the radio event observed on 12 July 2000 are presented in Figure 8 . The duration of 

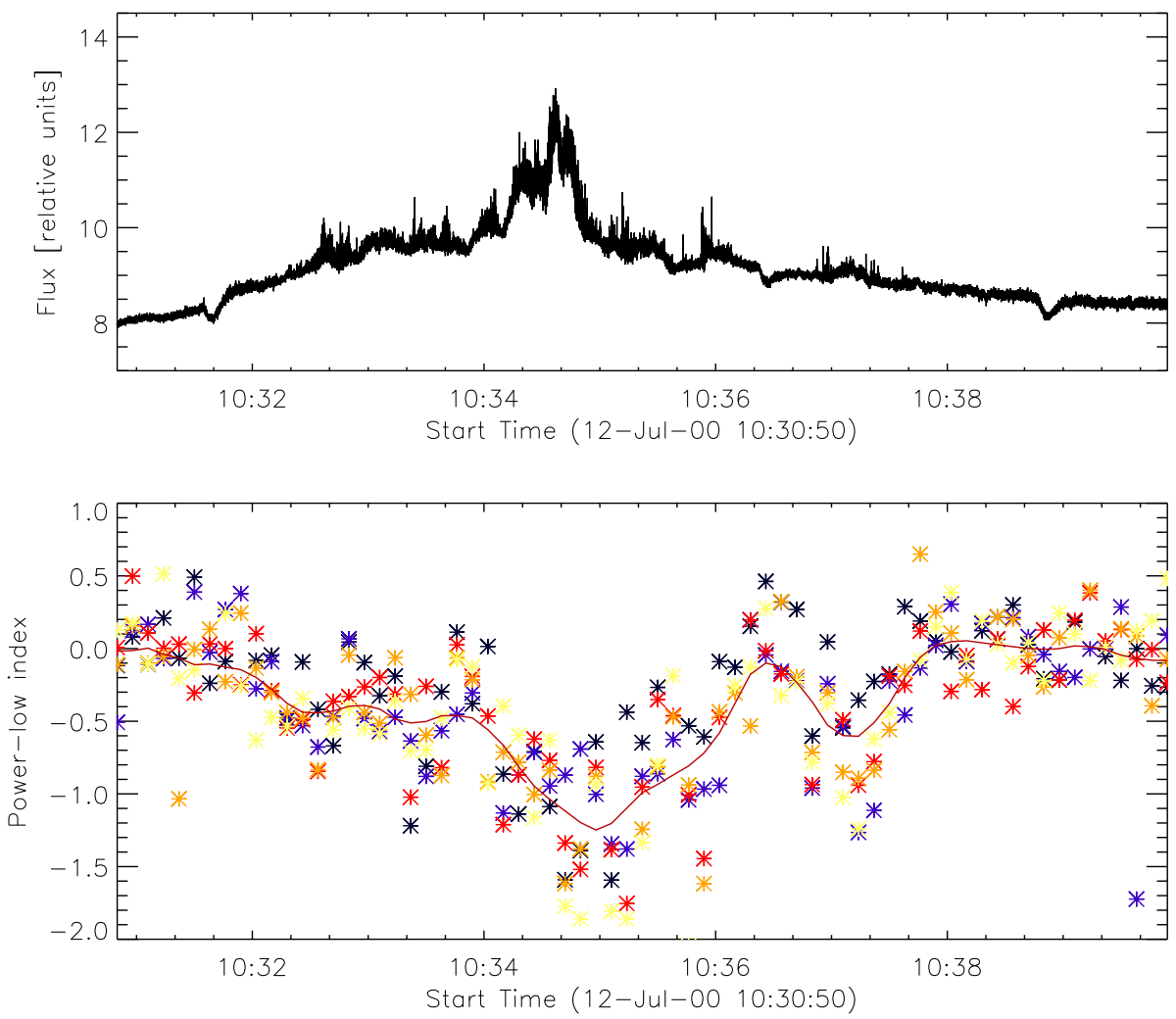

Figure 6 Drifting radio structure event recorded at Toruń on 12 July 2000 between 10:30:50 UT and 10:39:54 UT (upper panel). The Fourier spectral indices of the power spectra calculated for five adjacent frequency bands extending between 1434.5 MHz and 1446.5 MHz, $3 \mathrm{MHz}$ wide each (shown with colored stars). The red line shows double-smoothed values of momentary average Fourier spectral indices (lower panel).

the emission peaks was estimated at the half width of the Gaussian fittings. These durations ranged from $0.001 \mathrm{~s}$ up to $0.017 \mathrm{~s}$, while the mean duration is $0.008 \mathrm{~s}$.

\section{Discussion and Conclusions}

We presented an analysis of three radio events that included fine structures (two groups of dm-spikes and a DPS), observed on 15 March 2000, 21 June 2000, and 12 July 2000 during solar flares in the decimeter band from 0.8 to $2.0 \mathrm{GHz}$ at Ondřejov and in the $1352-$ $1490 \mathrm{MHz}$ band at Torun with very high time resolution (80 microseconds).

Cross-correlation coefficients of radio fluxes observed in adjacent frequency bands of the radio spectrum varied between 0.12 and 0.60 for dm-spikes and between 0.77 and 0.94 for the DPS event. The low values of the cross-channel correlation coefficients of the dm-spikes arise because their frequency bandwidths are more narrow than those of the DPS. Nevertheless, time variations of radio fluxes recorded even during the DPS event in the selected channels are similar, but still different. These differences can be explained by instrumental 
Figure 7 Examples of the power spectra of the eight-second-long data sets that include the investigated radio events. Upper panel: dm-spikes recorded in the 1407.5 MHz band at 12:20:38 UT on 15 March 2000. The Fourier spectral index is equal to -1.18 . Middle panel: dm-spikes observed in the $1431.5 \mathrm{MHz}$ band at 09:25:36 UT on 21 June 2000. The Fourier spectral index is equal to -0.92 . Lower panel: DPS event recorded at 10:35:06 UT on 12 July 2000 in the $1440.5 \mathrm{MHz}$ band. The Fourier spectral index is equal to -1.38 . All bands are $3 \mathrm{MHz}$ wide. Fourier spectral indices were estimated for $10-40 \mathrm{~Hz}$ segments of the power spectra (limited by blue vertical lines).
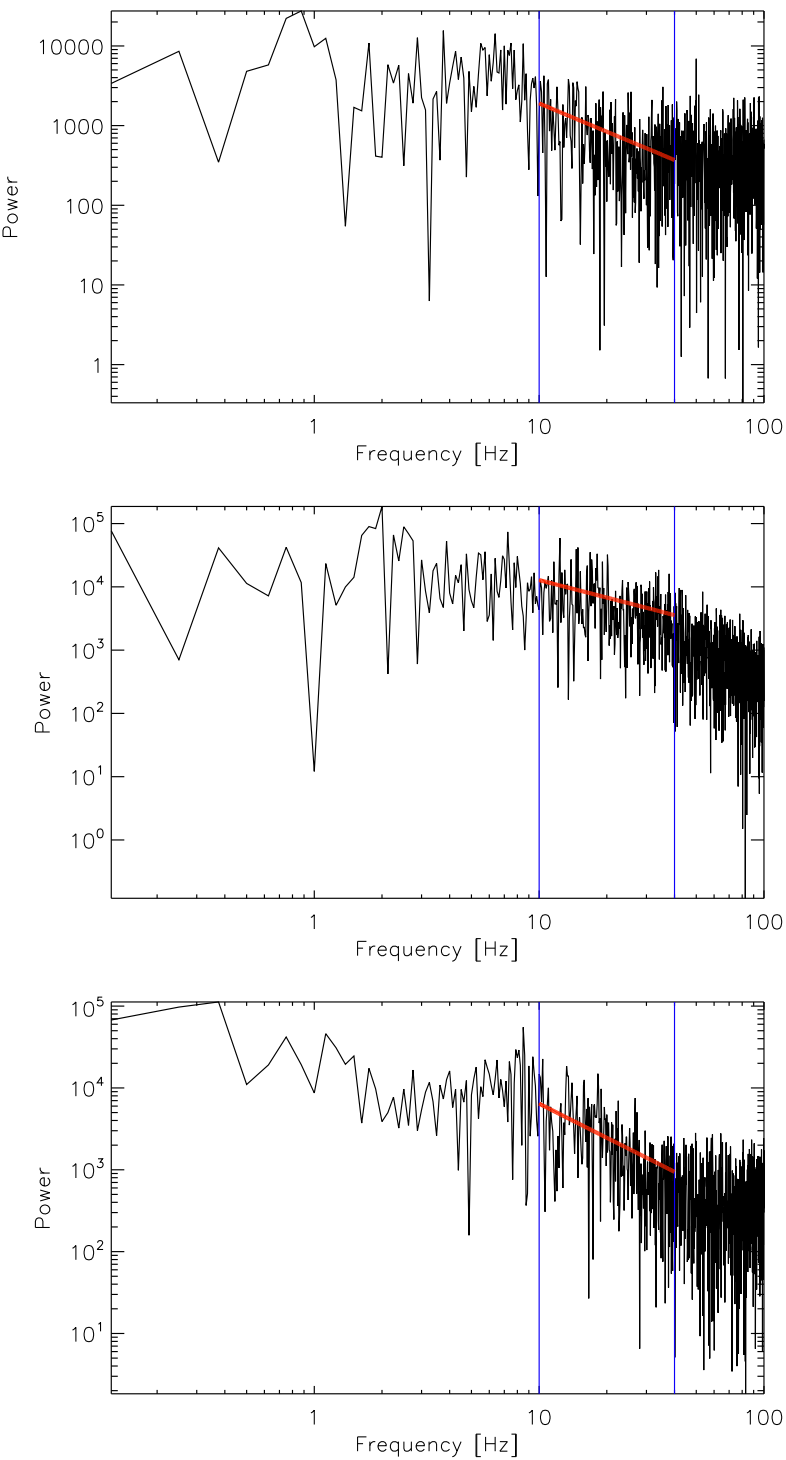

differences. However, assuming a plasma emission mechanism for the studied fine structures, the differences might be caused by actual physical reasons. In this case, the emission at each frequency is obviously generated at different densities and thus at different locations, in which the results of the physical processes can be different.

The Fourier spectra were calculated for a sequential series of intervals that lasted eight seconds each, which included all periods in which selected fine structures were observed. Five adjacent frequency channels were analyzed for each time interval. Thus, we simultaneously obtained the time evolution of the Fourier spectra and the time evolution of their Fourier spectral indices at five frequencies.

We found that the Fourier spectral indices varied rapidly during all these radio fine structures. However, at other times, the Fourier spectral indices remained close to zero, indicating 

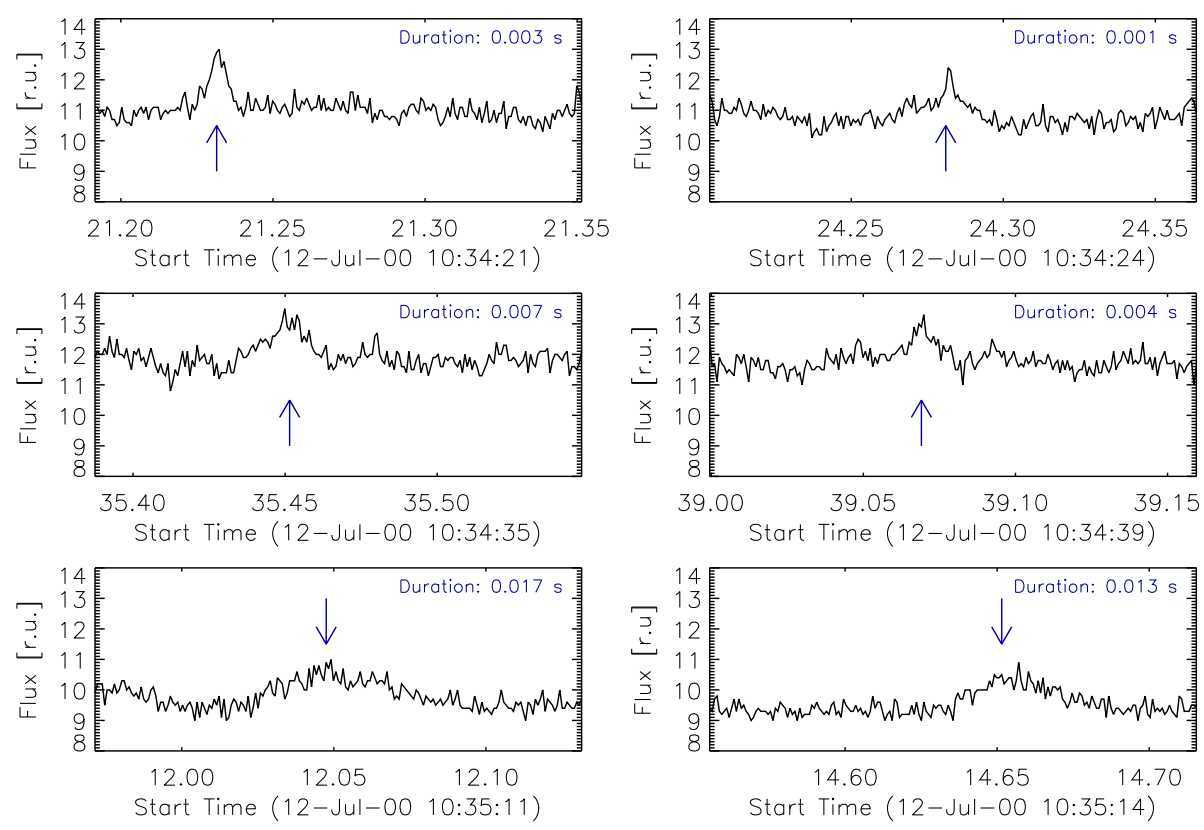

Figure 8 Examples of the shortest radio pulses (marked by vertical arrows) recorded during the DPS on 12 July 2000. Durations of the individual radio pulses are given in the upper right corners of each panel.

the presence of noise. For the $10-40 \mathrm{~Hz}$ frequency interval of the power spectra the mean values of the Fourier spectral indices varied from zero up to -1.51 for $\mathrm{dm}$-spikes and from zero up to -1.53 for the DPS event.

Furthermore, based on an extension of the Fourier spectra toward high spectral frequencies, we found that these fine structures are connected with very short processes with durations of up to $0.025 \mathrm{~s}$ (frequency $40 \mathrm{~Hz}$ ), and in some intervals even up to $\sim 0.01 \mathrm{~s}$.

The finding that the Fourier spectra of the dm-spikes have a power-law form is new. The similarity between the Fourier spectral indices of the dm-spikes and of the DPS shows that the DPS and narrowband dm-spikes might be physically similar. This supports the idea that these spikes are a radio signature of a cascade of interacting plasmoids of different sizes, as was previously proposed by Karlický, Bárta, and Jiřička (2013).

Therefore, in the following, we assume that these two fine structures are generated by a common plasma emission mechanism. This is supported by the general assumption that in coronal flare sources the electron plasma frequency is much higher than the electroncyclotron frequency.

While we interpret the DPS as the radio emission from one dominant plasmoid in accordance with the model by Kliem, Karlický, and Benz (2000), we interpret the dm-spikes as radio emission from a cascade of plasmoids (Karlický, Bárta, and Jiřička, 2013). Different sizes of these plasmoids cause bursts with not only different frequency bandwidths, but also different time scales. The time scales of these processes $t_{\text {scale }}$ can be expressed as the ratio of the spatial scales of plasmoids $l_{\mathrm{P}}$ to the local Alfvén speed $v_{\mathrm{A}}$.

It is expected that the plasmoid sizes in the cascade have a power-law distribution (Karlický and Bárta, 2011). The power-law Fourier spectra of the time variations of the studied DPS and dm-spikes meet this expectation. However, different Fourier spectral indices in different time intervals, even in neighboring channels (i.e. in different plasma densities 
corresponding to different heights in the flaring atmosphere) indicate that the plasmoid reconnection cascade is not in stationary state; on the contrary, it evolves very dynamically. Values of the Fourier spectral indices were in most cases different from the Kolmogorov spectral index $(-1.66)$, which is considered as the spectral index of MHD turbulence in stationary and saturated states. In relation to the DPS, our present results meet and support conclusions previously presented by Karlický et al. (2005).

The shortest individual emission structures found in the DPS event lasted only $0.001 \mathrm{~s}$. Assuming the Alfvén speed to be equal to $1000 \mathrm{~km} \mathrm{~s}^{-1}$, the relevant size of the plasmoid is of about $1 \mathrm{~km}$. This implies plasmoids that are much smaller than any plasmoid observed in UV and soft X-ray emissions.

Our work clearly shows that the high time resolution broadband radio spectral observations of the Sun can provide extremely valuable and unique data concerning physical processes occurring in the solar atmosphere and its subtle structure.

Acknowledgements This research was supported by the grant P209/12/0103 (GA CR), the project RVO:67985815 of the Astronomical Institute of the Academy of Sciences of the Czech Republic. Bartosz Dąbrowski would like to thank the staff and operators of the $15 \mathrm{~m}$ radio telescope of the Centre for Astronomy at the Nicolaus Copernicus University in Torun for their help in obtaining the data.

Open Access This article is distributed under the terms of the Creative Commons Attribution License which permits any use, distribution, and reproduction in any medium, provided the original author(s) and the source are credited.

\section{References}

Bárta, M., Büchner, J., Karlický, M., Skála, J.: 2011a, Spontaneous current-layer fragmentation and cascading reconnection in solar flares. I. Model and analysis. Astrophys. J. 737, 24.

Bárta, M., Büchner, J., Karlický, M., Kotrč, P.: 2011b, Spontaneous current-layer fragmentation and cascading reconnection in solar flares. II. Relation to observations. Astrophys. J. 730, 47.

Benz, A.O.: 1986, Millisecond radio spikes. Solar Phys. 104, 99.

Benz, A.O., Battaglia, M., Vilmer, N.: 2011, Location of decimetric pulsations in solar flares. Solar Phys. $273,363$.

Cadwell, B.J.: 1997, Pulsar survey and timing with the Penn State Pulsar Machines. PhD thesis, The Pennsylvania State University.

Dąbrowski, B.P., Rudawy, P., Karlický, M.: 2011, Millisecond radio spikes in the decimetric band. Solar Phys. 273, 377.

Dạbrowski, B.P., Rudawy, P., Falewicz, R., Siarkowski, M., Kus, A.J.: 2005, Millisecond radio spikes in the decimetre band and their related active solar phenomena. Astron. Astrophys. 434, 1139.

Fleishman, G.D., Yastrebov, S.G.: 1994, On the harmonic structure of solar radio spikes. Solar Phys. 154, 361.

Jiřička, K., Karlický, M., Kepka, O., Tlamicha, A.: 1993, Fast drift burst observations with the new Ondrejov radiospectrograph. Solar Phys. 147, 203.

Karlický, M.: 2004, Series of high-frequency slowly drifting structures mapping the flare magnetic field reconnection. Astron. Astrophys. 417, 325.

Karlický, M., Bárta, M.: 2011, Successive merging of plasmoids and fragmentation in a fare current sheet and their X-ray and radio signatures. Astrophys. J. 733, 107.

Karlický, M., Bárta, M., Nickeler, D.: 2012, Fragmentation during merging of plasmoids in the magnetic field reconnection. Astron. Astrophys. 541, A86.

Karlický, M., Bárta, M., Jiřička, K.: 2013, Fragmented reconnection and narrowband decimetric spikes. Cent. Eur. Astrophys. Bull. 37, 521.

Karlický, M., Fárník, F., Mészárosová, H.: 2002, High-frequency slowly drifting structures in solar flares. Astron. Astrophys. 395, 677.

Karlický, M., Jiřička, K., Sobotka, M.: 2000, Power-law spectra of $1-2$ GHz narrowband dm-spikes. Solar Phys. 195, 165.

Karlický, M., Sobotka, M., Jiřička, K.: 1996, Narrowband dm-spikes in the 2 GHz frequency range and MHD cascading waves in reconnection outflows. Solar Phys. 168, 375. 
Karlický, M., Bárta, M., Mészárosová, H., Zlobec, P.: 2005, Time scales of the slowly drifting pulsating structure observed during the April 12, 2001 flare. Astron. Astrophys. 432, 705.

Khan, J.I., Vilmer, N., Saint-Hilaire, P., Benz, A.O.: 2002, The solar coronal origin of a slowly drifting decimetric-metric pulsation structure. Astron. Astrophys. 388, 363.

Kliem, B., Karlický, M., Benz, A.O.: 2000, Solar flare radio pulsations as a signature of dynamic magnetic reconnection. Astron. Astrophys. 360, 715.

Kuijpers, J., van der Post, P., Slottje, C.: 1981, Runaway acceleration in a radio flare. Astron. Astrophys. 103, 331.

Kundu, M.R., Nindos, A., Vilmer, N., Klein, K.-L., Shibata, K., Ohyama, M.: 2001, Metric radio emission associated with X-ray plasmoid ejections. Astrophys. J. 559, 443.

Mészárosová, H., Veronig, A., Zlobec, P., Karlický, M.: 2003, Analysis of solar narrow band dm-spikes observed at 1420 and $2695 \mathrm{MHz}$. Astron. Astrophys. 407, 1115.

Shibata, K., Tanuma, S.: 2001, Plasmoid-induced-reconnection and fractal reconnection. Earth Planets Space 53, 473.

Tanuma, S., Yokoyama, T., Kudoh, T., Shibata, K.: 2001, Two-dimensional magnetohydrodynamic numerical simulations of magnetic reconnection triggered by a supernova shock in the interstellar medium: generation of X-ray gas in the galaxy. Astrophys. J. 551, 312. 